\title{
PLANEJAMENTO DA EXPANSÃO DE SISTEMAS DE TRANSMISSÃO DE ENERGIA ELÉTRICA COM BUSCA EM VIZINHANÇA VARIÁVEL
}

\author{
Silvia Lopes de Sena Taglialenha Rubén Romero ${ }^{2}$ \\ ${ }^{1}$ Departamento de Ciência Exatas - Universidade Federal de Matogrosso do Sul. ${ }^{2}$ Departamento de Engenharia Elétrica - Universidade \\ Estadual Paulista, Ilha Solteira, SP, BRASIL. E-mail: ruben@dee.feis.unesp.br, taglialenha@gmail.com
}

\section{RESUMO}

O problema de planejamento estático da expansão do sistema de transmissão de energia elétrica em longo prazo consiste em, dada uma configuração inicial e os dados de geração e de demanda do horizonte de planejamento, limites de operação, custos e restrições de investimento, determinar o plano de expansão com um custo mínimo, ou seja, deve-se determinar onde e que tipo de equipamentos devem ser instalados para que o sistema opere corretamente atendendo as necessidades do mercado de energia elétrica. É um problema do tipo não linear inteiro misto de elevada complexidade para sistemas de grande porte, apresentando o fenômeno de explosão combinatória e com o agravante de ser multimodal, sendo então muito complexo para ser resolvido através de técnicas tradicionais de otimização. Para resolver este problema aplicamos a metaheurística Busca em Vizinhança Variável, que está baseada num simples princípio: percorrer o espaço de busca (soluções possíveis) realizando trocas (diversificação) sistemáticas de estruturas de vizinhança dentro de um algoritmo de busca local (intensificação). Apresentamos resultados obtidos para redes de médio porte disponíveis na literatura, os quais mostram que esta metaheurística é robusta e competitiva em termos de qualidade e tempo de processamento.

Palavras-chave: busca através de vizinhança variável; planejamento da expansão de redes; metaheurísticas.

\section{ELECTRIC TRANSMISSION NETWORK EXPANSION PLANNING WHIT VARIABLE NEIGHBORHOOD SEARCH}

\section{ABSTRACT}

The static long-term power transmission network expansion planning problem consists in, given an initial configuration and the generation data and demand of the planning horizon, operation limits, costs and investment restrictions, to determine the expansion plan with a minimal cost, in other words, should be determined where and what type of equipments should be correctly installed for the system to operate assisting the needs of the electric power market. It is an integer nonlinear problem with high complexity for systems of great load, presenting the phenomenon of combinatorial explosion and with the added difficulty of being multimodal, then being too complex to be solved by traditional optimization techniques. To solve this problem we applied the metaheuristic Variable Neighborhood Search, which is based on a simple beginning: Systematic changes of neighborhood within a local search. We present obtained results using available nets of medium sized networks available in the literature, which show that this metaheuristic is robust and competitive both in terms of quality and processing time.

Keywords: variable neighborhood search; network expansion planning; metaheuristics. 


\section{INTRODUÇÃO}

O problema de planejamento da expansão de sistemas de potência, um problema de grande importância no setor elétrico, deve ser solucionado para garantir que os consumidores sejam atendidos de forma econômica e confiável com o aumento da demanda de energia ao passar dos anos. Apesar de o sistema elétrico estar passando por um processo de desregulamentação, transitando de uma estrutura centralizada para uma descentralizada, que tem como objetivo obter uma maior eficiência dos agentes participantes do setor, (agentes de geração, de transmissão, de distribuição, entre outros) que decidirão onde e quando investir seus recursos para expandir o sistema, este processo deverá sofrer a interferência de um agente central que deve funcionar como um plano de referência de forma a garantir uma expansão ótima global do sistema, otimizando a utilização dos recursos disponíveis e os custos para os consumidores. Novos parques geradores e novas rotas de transmissão devem ser construídos para atender esta nova carga do sistema.

O Brasil passou recentemente por um grave problema de racionamento de energia devido à falta de investimentos na área de geração e transmissão de energia, agravado por um período de seca que baixou o nível dos reservatórios. Como a maior parte da energia elétrica produzida no país é de origem hidrelétrica e as usinas estão distantes dos grandes centros consumidores, torna-se necessário a construção de novos circuitos de transmissão com a finalidade de transmitir a potência elétrica produzida nestas usinas, para aumentar a confiabilidade do sistema, otimizar recursos hídricos, etc.

As decisões do processo de planejamento estão relacionadas à seleção das melhores unidades geradoras, das melhores rotas de transmissão e das melhores malhas para garantir um suprimento de energia de forma econômica e confiável. Este processo de tomada de decisão dá origem a um problema de otimização complexo que deve ser resolvido pelos engenheiros de planejamento. É necessário o desenvolvimento de estratégias e técnicas de solução que assegurem que as decisões tomadas durante o processo de planejamento sejam as decisões ótimas. Este problema não pode ser resolvido sem que sejam feitas simplificações. Normalmente, o problema de planejamento é separado com relação aos seus principais agentes. O problema de planejamento da geração, que supõe conhecido o custo de expansão da transmissão, o problema de planejamento da transmissão, que supõe conhecidas estimativas de crescimento da demanda e programas alternativos de expansão da geração, até o ano horizonte de planejamento e o planejamento da distribuição.

No problema de planejamento da expansão de sistemas de transmissão (PPEST) ainda é possível separar o problema em dois tipos: planejamento estático e planejamento dinâmico. No planejamento estático existe apenas um estágio de planejamento, onde o planejador procura conhecer o circuito ótimo para ser adicionado em um único ano no horizonte de planejamento, ou seja, o planejador não está interessado em saber quando o circuito deverá ser instalado, mas encontrar a solução final ótima para uma futura situação definida. Por outro lado, se múltiplos anos são considerados e a estratégia de expansão ótima abrange todo o período, o planejamento é classificado como dinâmico. Neste caso, o modelo matemático deve conter restrições de tempo para considerar a expansão ao longo dos anos de tal forma que o valor presente dos custos ao longo do planejamento seja minimizado enquanto que as restrições impostas sejam verificadas. 
O planejamento dinâmico é muito complexo pois deve levar em consideração não só o tamanho e a alocação, mas também considerações sobre o tempo. Poucos trabalhos sobre planejamento dinâmico para problemas reais de planejamento podem ser encontrados na literatura. Em Latorre et al. (2003) são apontados alguns destes trabalhos.

Neste trabalho, estamos interessados no planejamento estático da expansão a longo prazo de sistemas de transmissão de energia elétrica (ROMERO et al., 2002; TAGLIALENHA; ROMERO, 2008), que consiste em encontrar o plano de expansão ótimo (construção de novos circuitos) para um horizonte de planejamento definido, ou seja, onde e que tipo de circuitos devem ser construídos para que o sistema opere adequadamente num horizonte de planejamento para um crescimento específico da demanda. É um problema muito complexo para ser resolvido através de técnicas tradicionais de otimização porque possui muitos ótimos locais, apresenta o fenômeno de explosão combinatória e ainda é do tipo não linear inteiro misto.

$\mathrm{Na}$ literatura especializada aparecem muitas propostas de solução do problema de planejamento de sistema de transmissão. Essas propostas podem ser separadas em dois grupos: i) métodos exatos de otimização, tais como os analíticos e os de otimização clássica (Decomposição de Benders e algoritmos Branch and Bound) (HAFFNER et al., 2001); e ii) métodos aproximados, como os algoritmos heurísticos e os metaheurísticos (Simulated Annealing (SA) (ROMERO et al., 1996), Genetic Algorithms (GA) (GALLEGO et al., 1998), Tabu Search (TS) (GALLEGO et al., 2000), GRASP (FARIA et al., 2005) etc. Entretanto, existem ferramentas que apresentam características destas duas categorias, e em geral também são chamadas de metaheurísticas.
Os métodos exatos, geralmente utilizando técnicas de decomposição matemática e técnicas de otimização clássica como programação linear, programação dinâmica, programação não linear e programação inteira mista apresentam a característica de que determinam a solução do problema de planejamento e são muito eficientes em sistemas de pequeno e médio porte, mas para sistemas de grande porte ainda apresentam problemas de convergência e de elevado esforço computacional.

Os métodos heurísticos são a alternativa atual para os modelos de otimização matemática. O termo 'heurística' é utilizado para descrever todas essas técnicas que, ao invés de usar otimização clássica, gera, passo a passo, uma solução avaliando e selecionando opções de expansão, com ou sem a ajuda do usuário. Para isto, as técnicas heurísticas realizam buscas locais com a orientação lógica ou empírica de índices de sensibilidade (critérios de qualidade). Estas regras são utilizadas para gerar e classificar as opções durante a busca. Em geral estes métodos apresentam a vantagem de fornecer soluções de boa qualidade com esforços computacionais pequenos, mas também não garantem que se encontre a solução ótima de sistemas reais. Entretanto, estes métodos ainda são os mais utilizados pelas concessionárias de energia elétrica nos trabalhos de planejamento.

Os algoritmos metaheurísticos, ferramentas surgidas mais recentemente, descrevem como se explorar o espaço de busca sem se prender a um problema específico. Estes algoritmos coordenam heurísticas mais simples, com uma busca local, com o propósito de encontrar soluções de melhor qualidade do que as obtidas utilizando as heurísticas isoladamente.

Neste trabalho desenvolvemos um algoritmo para resolver o PPEST utilizando a

\footnotetext{
1 Um método de resolver problemas através de técnicas práticas aprendidas em experiências passadas. Formada irregularmente da palavra grega 'heuriskein'.
} 
metaheurística Variable Neighborhood Search (VNS), que embora tenha sido apresentada em meados da década de 90, não se conhece, até o presente, nenhuma aplicação na solução deste problema. Proposta por Mladenović (1995) e Mladenović e Hansen (1997), é baseada num simples princípio: Explorar o espaço de soluções através de trocas sistemáticas de estruturas de vizinhança durante 0 processo de busca. Inicialmente, na Seção 2, apresentamos a definição e modelagem do PPEST, a estrutura básica deste método $e$ os detalhes da implementação da técnica em um sistema de médio porte disponível na literatura, cujos resultados aparecem na Seção 3, e finalmente, na Seção 4, apontamos algumas considerações sobre o método em relação a outras metaheurísticas.

$$
\text { Min } v=\sum_{(i, j) \in \Omega} c_{i j} n_{i j}
$$

s.a.

$$
\begin{aligned}
& S f+g=d \\
& f_{i j}-\gamma_{i j}\left(n_{i j}^{0}+n_{i j}\right)\left(\theta_{\mathrm{i}}-\theta_{j}\right)=0 \\
& \left|f_{i j}\right| \leq\left(n_{i j}^{0}+n_{i j}\right) \overline{f_{i j}} \\
& 0 \leq g \leq \bar{g} \\
& 0 \leq n_{i j} \leq \overline{n_{i j}} \\
& n_{i j} \text { inteiros } \\
& f_{i j}, \text { e } \theta_{j} \text { ilimitados, } \forall(i, j) \in \Omega
\end{aligned}
$$

\section{MATERIAL E MÉTODOS}

A modelagem matemática mais indicada para representar a operação adequada do PPEST seria por meio de relações matemáticas de fluxo de carga $A C$, tipicamente usada na análise da operação do sistema elétrico. Entretanto esta modelagem ainda não pode ser usada de forma eficiente no planejamento de sistemas de transmissão. Assim, a modelagem matemática mais indicada em trabalhos de planejamento de sistemas de transmissão é o chamado modelo DC (ROMERO et al., 2002) que leva em conta as duas leis de Kirchhoff apenas para o balanço e o fluxo de potência ativa, o qual pode ser assim formulado:
Em que, $c_{i j}, \gamma_{i j}, n_{i j}, n_{i j}^{0}, f_{i j}, \overline{f_{i j}}$ e $\theta_{i}$ representam, respectivamente, o custo de um circuito que pode ser adicionado no caminho $i-j$, a susceptância do circuito, o número de circuitos adicionado no caminho $i-j$, o número de circuitos presentes na configuração base, o fluxo de potência, o fluxo máximo no caminho $i-j$, e a magnitude do ângulo de tensão na barra $i$. O investimento é dado por $v, S$ é a matriz transposta do sistema elétrico, $f$ é um vetor com componentes $f_{i j}$, $g$ é um vetor com componentes $g_{k}$ (geração na barra $k$ ) cujo valor máximo é $\bar{g}, \bar{n}_{i j}$ é o número máximo de circuitos que podem ser adicionados no caminho $i-j$, e $\Omega$ é o conjunto formado pelos índices dos circuitos candidatos à adição.

O conjunto de restrições (2.2) representa as equações correspondentes à primeira lei de Kirchhoff, uma equação para cada barra do sistema, e as restrições (2.3) representa as equações correspondentes à segunda lei de 
Kirchhoff. As restrições (2.4) representam as restrições de capacidade de transmissão dos circuitos (linhas e/ou transformadores) e o valor absoluto é necessário porque os fluxos de potência podem fluir nos dois sentidos. As restrições (2.5) e (2.6) são triviais e representam apenas restrições de limite de geração e de circuitos adicionados em cada caminho candidato $i-j$. Finalmente, as variáveis $f_{i j}$ são irrestritas em valor e as variáveis $n_{i j}$ devem ser inteiras, representando a maior fonte de complexidade no problema, ou seja, o problema resultante é do tipo de programação não linear inteiro misto de elevada complexidade no processo de solução para sistemas de grande porte.

Uma modelagem mais simplificada é o chamado modelo de transportes que considera apenas a primeira lei de Kirchhoff, resultando num problema do tipo linear inteiro misto (VILLASANA et al., 1985). Mesmo sendo linear, ainda não é possível encontrar a solução ótima para o modelo de transportes para sistemas de grande porte e de elevada complexidade. Neste trabalho utilizamos 0 modelo de transportes embora a proposta possa ser facilmente

que a cada solução $x \in X$ associa um conjunto de soluções $N(x) \subset S$, denominada vizinhança de $x$.

Denotemos por $N_{k}, k=1, \ldots, k_{\max }$, um conjunto finito pré-selecionado de estruturas de vizinhança e por $N_{k}(x)$, o conjunto de soluções na $k$-ésima vizinhança de $x$. As vizinhanças $N_{k}(x)$ podem ser induzidas por uma ou mais métricas (ou quase-métricas) introduzidas num espaço de solução $S$. Uma solução ótima $x_{o p}$ (ou mínimo global) é uma solução onde o mínimo de (2.1.a) é alcançado. Dizemos que $x^{\prime}$ é um estendida para o modelo DC já que estamos utilizando uma metaheurística, como pode ser visto em Taglialenha e Romero (2006; 2008).

\subsection{Estrutura básica da ferramenta VNS}

Consideremos um problema de otimização combinatória representado da seguinte forma: $\operatorname{Min}\{f(x): x \in X, X \subseteq S\}$, em que $S$ é o espaço de soluções, $X$ é o conjunto de soluções factíveis, $x$ é uma solução alternativa e $f$ é uma função que assume valores reais.

A VNS, ao contrário de muitas metaheurísticas baseadas em busca local, não segue uma trajetória de busca, mas explora a partir de uma solução inicial, uma seqüência crescente de vizinhanças distantes, e só realiza o movimento até uma nova solução se esta solução for melhor que a solução incumbente (HANSEN; MLADENOVIĆ, 2003a; 2003b).

Uma estrutura de vizinhança no espaço de soluções $S$ é uma aplicação

$N: X \rightarrow 2^{S}$,

mínimo local de (2.1.a) com relação a $N_{k}(x)$, se não existir solução $x \in N_{k}(x) \subseteq X$ tal que $f(x)<f\left(x^{\prime}\right)$.

Em geral, uma busca local de descida troca a solução atual por outra solução melhor na vizinhança considerada, portanto apresenta o risco de estacionar num mínimo local. As metaheurísticas baseadas em busca local aplicam distintas formas de continuar a busca depois de encontrar um ótimo local. A VNS baseia-se em três fatos simples: 1- Um mínimo local em relação a uma estrutura de vizinhança não é necessariamente um mínimo local com relação a uma outra; 2- Um mínimo global é um 
mínimo local em relação a todas as estruturas possíveis de vizinhança; 3- Para muitos problemas, um mínimo local em relação a uma ou a várias vizinhanças $N_{k}(x)$, são relativamente próximos um do outro.

Esta última observação, a qual é empírica, implica que um ótimo local sempre fornece alguma informação do ótimo global. Estes fatos sugerem então o uso de várias estruturas de vizinhança nas buscas locais para abordar um problema de otimização, como pode ser visto em Hansen et al. (2003) e Mladenović e Hansen (1997). E a troca de estrutura de vizinhança pode então ser realizada de forma determinística, aleatória, ou determinística e aleatória.

\subsubsection{VNS de Descida}

Uma busca local que determina a melhor solução na vizinhança da solução atual, a busca clássica de descida Variable Neighborhood Search Descent (VND) (MLADENOVIĆ, 1995), consiste em trocar, iterativamente, a solução incumbente pelo resultado da busca local desde que a nova solução seja melhor que a incumbente. Se a troca de estrutura de vizinhança é realizada de forma determinística cada vez que se chega a um ótimo local, se obtém a VND (MLADENOVIĆ, 1995; MLADENOVIĆ; HANSEN, 1997).

A solução final proporcionada por este algoritmo é um mínimo local em relação a todas as $k_{\max }$ estruturas de vizinhança, e, portanto a chance de se alcançar um mínimo global é maior de que quando se usa somente uma única estrutura.

Da ordem seqüencial das estruturas de vizinhança na VND pode se desenvolver uma estratégia aninhada. Por exemplo, se $k_{\max }=3$ então uma possível estratégia aninhada é executar a VND descrita acima para as duas primeiras estruturas de vizinhança em cada $x^{\prime}$ que pertença à terceira vizinhança de $x^{\prime}$, isto é, $\forall x \in N_{3}(x)$. Aplicações da VND podem ser encontradas em Belacel et al. (2002), Brinberg et al. (2000) e Hansen e Mladenović (2001b).

Os passos da VND são descritos a seguir.

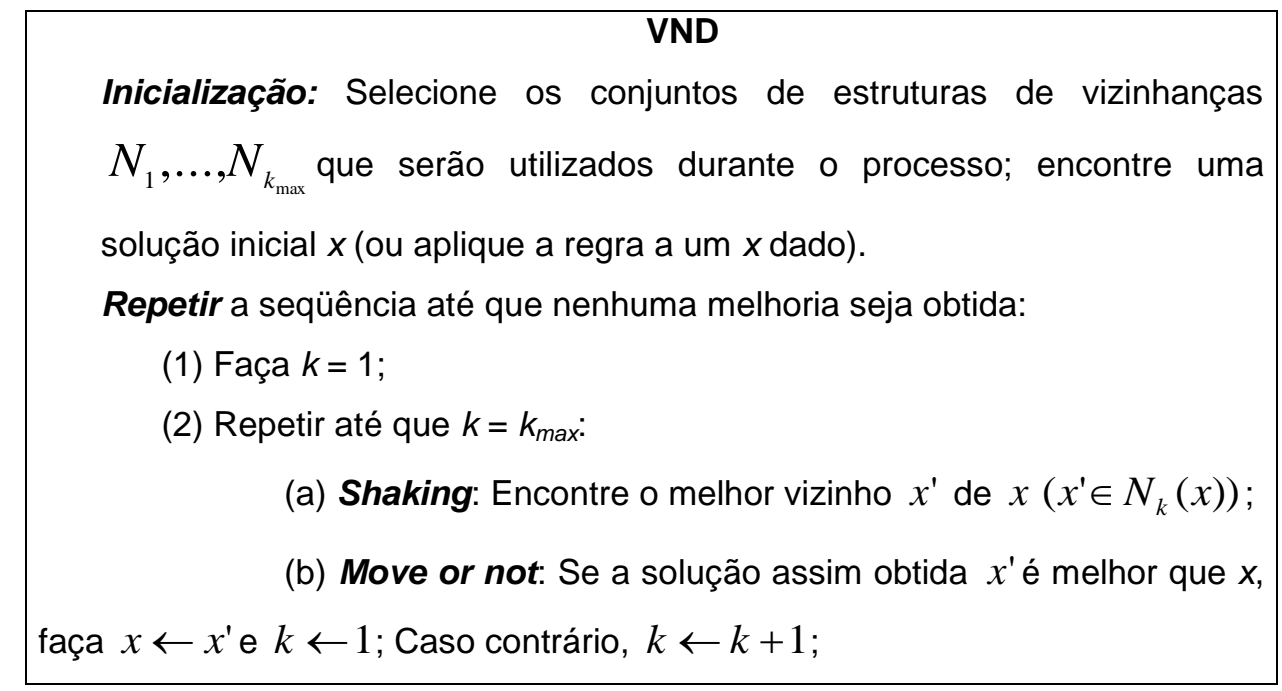

\subsubsection{VNS Básica}

A busca em vizinhança variável básica (Basic Variable Neighborhood Search, VNS)
(HANSEN; MLADENOVIĆ, 2003a) combina trocas determinísticas e aleatórias de estruturas de vizinhança. Os passos da VNS estão descritos 
a seguir, em que a condição de parada pode ser, por exemplo, o número máximo de iterações, ou o número máximo de iterações entre duas melhoras.

\section{VNS}

Inicialização: Selecione os conjuntos de estruturas de vizinhanças $N_{1}, \ldots, N_{k_{\max }}$ que serão usados durante o processo; encontre uma solução inicial $x$; escolha um critério de parada;

Repetir a seqüência até que a condição de parada esteja satisfeita:

(1) Faça $k \leftarrow 1$;

(2) Repetir até que $k=k_{\max }$ :

(a) Shaking: Gere um ponto $x^{\prime}$ aleatório em $N_{k}(x)$;

(b) Local search: Aplique algum método de busca local usando $x^{\prime}$ como solução inicial; denote por $x^{\prime \prime}$ a solução encontrada;

(c) Move or not: Se este ótimo local é melhor que a incumbente, faça $x \leftarrow x^{\prime \prime}$ e continue a busca em $N_{1}(k \leftarrow 1)$; Caso contrário, $k \leftarrow k+1$;

Observa-se que a solução $x^{\prime}$ é gerada aleatoriamente no passo (2a) para evitar ciclagem, o que pode ocorrer se for usada uma regra determinística qualquer.

A VNS foi aplicada com grande êxito em muitos problemas de otimização combinatória, entre os quais se encontram os problemas mais relevantes de planejamento logístico: o problema de empacotamento (FLESZAR; HINDI, 2002), de localização e de rotas. Ademais também foram abordados problemas onde as soluções são conjuntos de arestas, como as árvores, e alguns problemas de otimização contínua. Este problema foi abordado em Hansen e Mladenović (1997) e Hansen et al. $(2001$; 2003) utilizando a VNS básica e outras variantes da VND. Diversas variantes da VNS foram aplicadas em Brinberg et al. (2000) ao problema de janelas múltiplas, que é uma versão contínua do problema das $p$-medianas, onde as $p$ localizações podem ser escolhidas em todo o plano, onde também estão localizados os usuários. Outro tipo de problema de otimização combinatória importante, em relação a todo contexto de planejamento logístico, são os problemas de rotas. Tanto as versões clássicas do problema do caixeiro viajante (Traveling Salesman Problem), do problema de rota de veículos (Vehicle Routing Problem), como algumas de suas extensões foram abordadas com a VNS (HANSEN; MLADENOVIĆ, 1997; MLADENOVIĆ; HANSEN, 1997).

\subsection{VND no PPEST}

Nesta seção são apresentadas as diferentes etapas da VND aplicada ao PPEST.

\subsubsection{Solução inicial}

Para gerar a solução inicial utilizaremos um algoritmo heurístico construtivo (AHC), ou seja, um procedimento iterativo de escolhas ou decisões tomadas passo a passo que determina uma solução de boa qualidade de um problema complexo. No caso do PPEST, em cada passo é escolhido um circuito a ser adicionado ao sistema, sendo que a escolha é dada por um 
indicador de sensibilidade especificado pelo AHC. O processo iterativo é finalizado quando uma solução factível e geralmente de boa qualidade foi encontrada.

Embora os AHC sejam robustos e convirjam rapidamente, em problemas grandes e complexos apenas encontram soluções locais de

$$
\begin{array}{ll}
\text { Min } v= & \sum_{(i, j) \in \Omega} c_{i j} n_{i j} \\
\text { s.a. } & \\
& S f+g=d \\
& \left|f_{i j}\right| \leq\left(n_{i j}+n_{i j}^{0}\right) \bar{f}_{\mathrm{ij}} \\
& 0 \leq g \leq \bar{g} \\
& 0 \leq n_{i j} \leq \overline{n_{i j}} \\
& n_{i j} \text { inteiro, } f_{i j} \text { irrestrito }
\end{array}
$$

\section{Algoritmo Heurístico de Garver (AHG)}

A proposta de Garver (1970) consiste em resolver o sistema (2.2.1.a) relaxando a integralidade das variáveis inteiras $n_{i j}$, e encontrar a solução ótima contínua para a configuração corrente $n_{i j}^{0}$, em que $n_{i j}$ são os circuitos adicionados no processo iterativo do AHG. Assim, conhecidas as incógnitas $n_{i j}$ de (2.2.1.a) encontradas resolvendo um algoritmo de $\mathrm{PL}$, podemos encontrar os fluxos de potência em todos os circuitos da topologia corrente $\left(n_{i j}^{0}\right)$ e pelos circuitos (geralmente não inteiros) adicionados pelo $\mathrm{PL}\left(n_{i j}\right)$. Portanto, aquele caminho em que $n_{i j} \neq 0$ e que leva o maior fluxo de potência representa o caminho mais atrativo de acordo com esta proposta.

Um processo repetitivo desta estratégia, adicionando em cada passo um circuito no caminho mais atrativo, constitui o AHG. O processo termina quando a solução do $\mathrm{PL}$ correspondente à configuração corrente apresenta uma solução com todos os $n_{i j}=0$, o que significa que não é mais necessário realizar boa qualidade que podem diferenciar-se muito do ótimo global.

Apresentamos um AHC utilizando 0 Modelo de Transportes misto (VILLASANA et al., 1985) em que apenas as restrições da primeira lei de Kirchhoff, devem ser obrigatoriamente satisfeitas, o qual é dado por:

adições e o conjunto de circuitos adicionados representa a proposta de solução do algoritmo de Garver.

O algoritmo heurístico construtivo de Garver pode ser resumido nos seguintes passos:

1. Assumir a configuração base $n_{i j}^{0}$ como configuração corrente.

2. Resolver o PL correspondente do sistema (2.2.1.a) para a configuração corrente. Se todos os $n_{i j}=0$ então pare, pois foi encontrada uma boa configuração factível. Em caso contrário ir ao passo 3.

3. Calcular os fluxos através de todos os novos circuitos adicionados pelo $\mathrm{PL},\left(n_{i j} \neq 0\right)$, usando a relação $f_{i j}^{v}=n_{i j} \bar{f}_{i j}$. Identificar o novo caminho $i j$ com o maior valor de $f_{i j}^{v}$ e atualizar a configuração corrente adicionando um circuito naquele caminho $i j$. Voltar ao passo 2.

Do ponto de vista de otimização matemática o $\mathrm{AHG}$ é um algoritmo heurístico construtivo que na prática encontra configurações de boa qualidade, mas do ponto de vista teórico, 
não existe garantia de encontrar a solução ótima global. Detalhes podem ser vistos em Taglialenha e Romero (2008).

\subsubsection{Definição das Vizinhanças}

Dada uma solução $x$, utilizando a definição (2.1.a), podemos definir a seguinte estrutura de vizinhanças no espaço de solução $S:$

$$
N_{k}(x)=\left\{x^{\prime} \in X: d\left(x, x^{\prime}\right)=k, k=1, \ldots, k_{\max }\right\},
$$

(a)

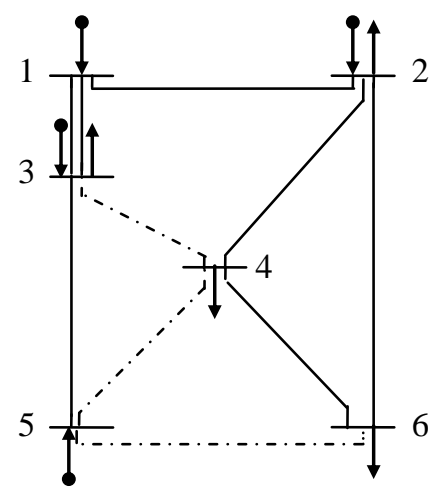

(b)

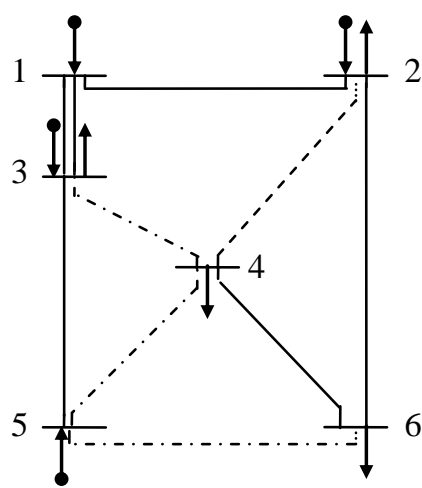

(c)

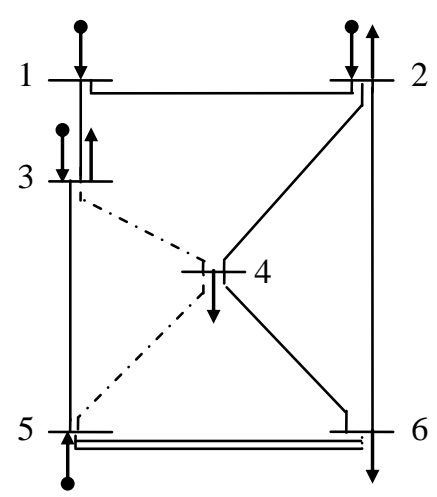

Figura 1. Sistema de 6 barras.

Para exemplificar, consideremos 0 sistema de seis barras da Figura 1(a) com sete circuitos na configuração base, que é codificado conforme $x$ na Figura 2.

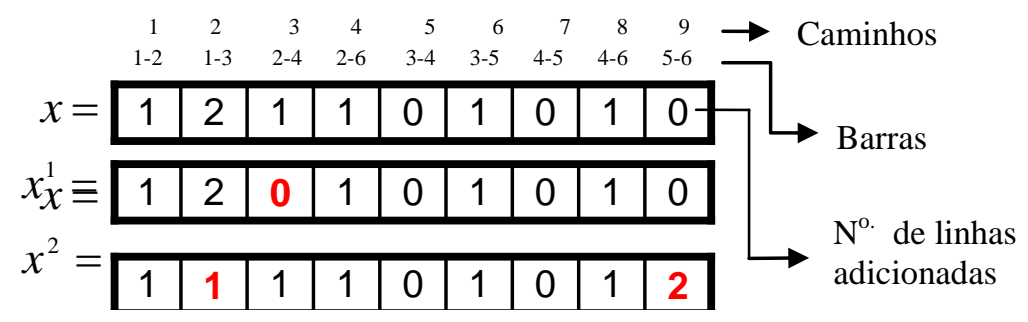

Figura 2. Vizinhos em $N_{1}(x)$ e $N_{2}(x)$.

A configuração $x^{1}$ da Figura 2 é vizinho de $x$ em $N_{1}(x)$ e representa o sistema da Figura1(b), e $x^{2}$ é vizinho de $x$ em $N_{2}(x)$, representando o sistema da Figura 1(c). O vizinho $x^{1} \in N_{1}(x)$ foi obtido a partir de $x$ com a retirada de um circuito no caminho 3 (entre as barras 2 e 4), e o vizinho $x^{2} \in N_{2}(x)$ foi obtido a partir de $x$ com a retirada de um circuito no caminho 2 (entre as barras 1 e 3 ) e com a adição de dois circuitos no caminho 9 (entre as barras 5 e 6). Da mesma forma podemos obter os vizinhos nas demais vizinhanças.

\subsubsection{Busca local}

A partir da configuração inicial $x$ determinada pelo $A H G$, inicia-se a busca local em $N_{1}(x)$, ordenando os circuitos por ordem decrescente de custo e tentando retirar aqueles que, uma vez simulada sua saída (resolvendo o 
PL (2.2.3.a)), não produzam corte de carga no sistema. Assim, deve-se apenas verificar se com a proposta de solução o sistema opera com corte

$$
\begin{aligned}
& \text { Min } \omega=\sum_{k \in \Omega} r_{k} \\
& \text { s.a. } \\
& \quad S f+g+r=d \\
& \quad\left|f_{i j}\right| \leq\left(n_{i j}+n_{i j}^{0}\right) \bar{f}_{\mathrm{ij}} \\
& \quad 0 \leq g \leq \bar{g} \\
& \quad 0 \leq r \leq \bar{d} \\
& f_{i j} \text { irrestrito, } \forall i j \in \Omega
\end{aligned}
$$

em que $\omega$ representa 0 corte de carga do sistema e os $r_{k}$ representam os cortes de carga nas barras de demanda, que é equivalente a geração artificial. Se $\omega=0$, significa que a topologia avaliada representa uma solução factível para o modelo de transportes. Em caso contrário, a proposta representa uma solução infactível e deve ser descartada.

Nas demais vizinhanças, a partir da solução incumbente determinada na vizinhança anterior, geram-se os vizinhos respectivos, e calculam-se as variações de custos devido as trocas de linhas (diferença do custo dos circuitos que entraram e circuitos que saíram) e só foram simuladas as trocas (resolvendo o PL (2.2.3.a)) para linhas que apresentaram variação negativa. Se a simulação apontar uma configuração factível, ou seja, $\omega=0$ em (2.2.3.a), ela é então efetivada com a atualização da configuração corrente. No caso de a configuração ser infactível, a simulação é cancelada. Cabe esclarecer aqui que o movimento só é realizado se a nova configuração é melhor que a incumbente.

\section{RESULTADOS OBTIDOS}

O algoritmo proposto foi implementado utilizando a linguagem de programação FORTRAN, e o MINOS 5.0 como uma sub-rotina ou sem corte de carga, o que é feito resolvendose o seguinte PL:

de resolução de PL no sistema Sul Brasileiro. Este sistema apresenta 46 barras, 79 circuitos e uma demanda de 6880 MW. É possível a execução do planejamento com (CR), ou sem (SR) re-programação da geração. Os dados do sistema estão disponíveis em Haffner et al. (2001) e ROMERO et al. (2002).

O algoritmo implementado alcançou o ótimo global (HAFFNER et al., 2001), para os dois casos considerados.

Planejamento com reprogramação: Investimento: $v=$ US $\$ 53,334,000$, resolvendo 2,263 PL's, com as seguintes adições: $n_{20-21}=1$, $n_{20-23}=1, n_{42-43}=1, \quad n_{05-11}=2$ e $\quad n_{05-06}=1$, passando pelas seguintes estruturas de vizinhança: $N_{1}, N_{2}, N_{1}, N_{2}, N_{3}$.

Planejamento sem reprogramação: Investimento $v=$ US $\$ 127,272,000 \quad n_{14-16}=1$, $n_{18-19}=1, \quad n_{20-21}=1, \quad n_{42-43}=2, \quad n_{05-11}=2$, $n_{25-32}=1, \quad n_{31-32}=1, \quad n_{28-31}=1$, $n_{46-11}=1, n_{24-25}=2$, resolvendo 940,135 PL's, passando pelas seguintes estruturas de vizinhança:

$N_{1}, N_{1}, N_{2}, N_{3}, N_{4}, N_{1}, N_{1}, N_{2}, N_{3}, N_{4}, N_{1}, N_{2}, N_{3}, N_{4}, N_{5}$ e $N_{6}$.

Observemos que no caso do planejamento sem reprogramação da geração, se 
depois da segunda busca local em $N_{4}$ tivéssemos feito $k=6$, teríamos a convergência com pelo menos 670,000 PL's a menos do que se fazendo $k=1$.

\section{CONCLUSÕES}

O algoritmo proposto apresentou resultados excelentes na solução do PPEST.

Um ponto crítico na estratégia de busca em vizinhanças aplicada no PPEST é a escolha da estrutura da vizinhança, ou seja, a maneira pela qual a vizinhança é definida, pois o número de vizinhos a serem analisados cresce exponencialmente com a dimensão do problema.

É uma metaheurística que conta com uma extensa literatura, o que demonstra seu alto interesse, tanto teórico como prático, em várias áreas de otimização.

\section{REFERÊNCIAS}

BELACEL, N.; HANSEN, P.; MLADENOVIĆ, N. Fuzzy J-means: a new heuristic for fuzzy clustering. Pattern Recognition, v.35; n.10, p.2193-2200, 2002.

BRINBERG, J.; HANSEN, P.; MLADENOVIC, N.; TAILLARD, É. Improvements and comparison of heuristics for solving the multisource weber problem. Operations Research, v.48, n.3, p.444460, 2000.

FARIA, H. J.; BINATO, S.; RESENDE, M. G. C.; FALCÃO, D. M. Power transmission network design by greedy randomized adaptive path relinking. IEEE Transaction on Power System, v.20, n.1, p.43-49, 2005.

FLESZAR, K.; HINDI, K. S. New heuristics for one-dimensional bing-packing. Computers Operations Research, v.29, n.7, p.821-839, 2000.

GALLEGO, R. A.; MONTICELLI, A.; ROMERO, R. Transmission expansion planning by extended genetic algorithm. Proc. Inst. Elect. Eng.-Gen., Transm. Dist., v.145, n.3, p.329-335, 1998.

GALLEGO, R. A.; MONTICELLI, A.; ROMERO, R. Tabu Search algorithm for network synthesis. IEEE Transaction on Power System, v.15, n.2, p.490-495, 2000.
GARVER, L. L. Transmission network estimation using linear programming. IEEE Transaction Apparatus Systems, v.89, p.1688-1697, 1970.

HAFFNER, S.; MONTICELLI, A.; GARCIA, A.; ROMERO R. Specialized branch-and-bound algorithm for transmission network expansion planning. IEE Proceedings - Generation, Transmission and Distribution, v.148, n.5, p.521-526, 2001.

HANSEN, P.; MLADENOVIĆ, N. Variable neighborhood search for the $p$-midiam. Location Science, v.5, p.207-226, 1997.

HANSEN, P.; MLADENOVIĆ, N. Variable neighborhood search: Principles and applications. European Journal of Operational Research, v.130, p.449-467, (2001a).

HANSEN, P.; MLADENOVIĆ, N. J-means: a new local search heuristic for minimum sum-ofsquares clustering. Pattern Recognition, v.34, n.2, p.405-413, (2001b)

HANSEN, P.; MLADENOVIĆ, N. A tutorial on variable neighborhood search. Les Cahiers du GERAD, 2003a . G-2003-46.

HANSEN, P.; MLADENOVIĆ, N. Variable neighborhood search. In: Handbook of Metaheuristics. Kluwer, 2003b. cap. 6.

HANSEN, P.; MLADENOVIĆ, N.; PÉREZ, J. A. M. Variable neighborhood decomposition search. Journal of Heuristic, v.7, n.4, p.335-350, 2001.

HANSEN, P.; MLADENOVIĆ, N.; PÉREZ, J. A. M. Variable neighborhood Search. Revista Iberoamericana de Inteligência Artificial, n.19, p.7792, 2003.

LATORRE, G.; CRUZ, R. D.; AREIZA, J. M.; VILLEGAS A. Classification of publication and model on transmission expansion planning. IEEE Transaction on Power Systems, v.18, n.2, p.938-946, 2003.

MLADENOVIĆ, N. A variable neighborhood algorithm - a new metaheuristic for combinatorial optimization. Abstracts of Papers Presented at Optimization Days, p. 112, 1995.

MLADENOVIĆ, N.; HANSEN, P. Variable neighborhood search. Computers Ops. Research, v.24, n.11, p.1097-1100, 1997.

ROMERO, R.; GALLEGO, R. A.; MONTICELLI, A. Transmission system expansion planning by simulated annealing. IEEE Trans. Power Syst., v.11, p.364-369, 1996. 
ROMERO, R.; MONTICELLI, A.; GARCIA, A.; HAFFNER, S. Test systems and mathematical models for transmission network expansion planning. IEE Proceedings Generation, Transmission and Distribution, v.149, n.1, p.2736, 2002.

TAGLIALENHA, S. L. S; ROMERO, R. Busca em vizinhança variável para 0 problema de planejamento da expansão do sistema de transmissão de energia elétrica. In: XXXVIII Simpósio Brasileiro de Pesquisa Operacional. Anais... Goiânia, 2006.

TAGLIALENHA, S. L. S; ROMERO, R. Novas aplicações de metaheurísticas na solução do problema de planejamento da expansão do sistema de transmissão de energia elétrica. 2008. Tese (Doutorado), DEE-FEIS-UNESP, Ilha Solteira.

VILLASANA, R.; GARVER, L.L.; SALON, S.J. Transmission network planning using linear programming. IEEE Transaction on Power Apparatus and Systems, 1985. PAS-104(2). 